\title{
Authentication Issues in Multi-service Residential Access Networks
}

\author{
Judith Rosseb $\emptyset^{1}$, John Ronan ${ }^{2}$, and Kristian Walsh ${ }^{3}$ \\ 1 Telenor Research \& Development, Telenor Communication II AS, \\ N-1331 Fornebu, Norway, judith.rossebo@telenor.com \\ 2 Telecommunications Systems Software Group, \\ Waterford Institute of Technology, Waterford, Ireland, jronan@tssg.org \\ 3 Department of Mathematics and Computing, \\ Cork Institute of Technology, Cork, Ireland, krwalsh@cit.ie
}

\begin{abstract}
Multi-service residential access networks allow residential customers to choose amongst a variety of service offerings, over a range of Core Networks and subject to user requirements such as QoS, mobility, cost and availability. These issues place requirements on authentication for network access, with a need for mutual authentication of the residential gateway ( $R G$ ) to the local access point (LAP). The EU-IST project TORRENT is building a testbed providing for multiservice residential access networks in order to demonstrate the benefit of intelligent control, both for the customer and for the network operators and service providers. Adequate security measures are essential in order to secure access to the TORRENT system and services and for QoS provisioning to authorised users. This paper examines the authentication issues for the TORRENT system and presents a public key based authentication protocol for mutually authenticating the RG and the LAP.
\end{abstract}

Keywords: Authentication, Public Key Infrastructure, Encryption, Residential Gateway

\section{Introduction}

MULTI-SERVICE RESIDENTIAL access networks are concerned with exploiting the use of shared physical access networks for a range of different services and traffic types optimising bandwidth utilisation in existing access networks while meeting user requirements related to QoS, security, cost and availability. In order to deliver such offerings to the residential customers, an infrastructure is required mapping user requirements to the appropriate networks, services, and applications. The infrastructure typically incorporates a residential gateway in the home and one or more serving local access point(s)(LAP) integrating access technologies with services and core networks. The residential gateway(RG) connects the home network technologies (especially WLAN, Ethernet) to the access network(s), e.g. cable, ISDN, $x$ DSL, wireless, LMDS or any combination thereof. 
Functions of the local access point may include providing customer negotiation facilities and host accounting and security functionality (e.g., AAA services) of customer access to e.g., metering, security, and monitoring.

This work has evolved from investigations in the TORRENT project of authentication issues in multi-service residential access networks. In future multiservice residential access networks, users may have the opportunity to choose between several network operators and service providers. Authentication requirements of the different network operators and service providers can lead to the situation that a user has to keep track of several different usernames and passwords and different methods of authenticating to the networks and services. There is a need for a single mechanism for authentication, which gives the user access to all services. For this, the mechanism should be suitable for services requiring strong authentication (e.g. signing your loan application electronically). This paper discusses the issues involved and presents our scheme for publickey based authentication for which a smart card is used as certificate and key container and may also be used for authentication to many services.

\subsection{TORRENT Overview}

TORRENT [8] is an EU-supported Framework V project, aiming to build a testbed for multi-service residential access networks. This test-bed (Figure 1) will allow demonstration of the benefit of intelligent control for the customer, network operators and service providers. An important goal is to optimise the bandwidth utilisation in existing access and core networks, while at the same time meeting user requirements in an optimal manner. These requirements include Quality of Service (QoS), security, cost, and availability. Security is of major importance, and adequate security measures are essential in order to secure access to the TORRENT system and services, and also for provisioning QoS to authorised users.

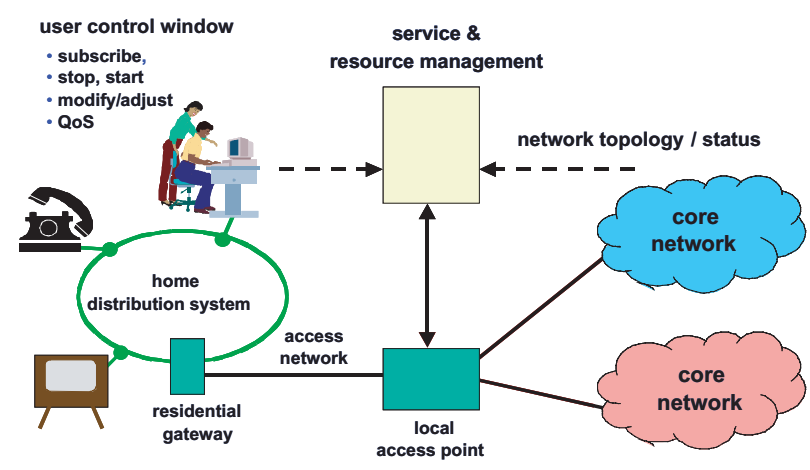

Fig. 1. TORRENT Architecture 
IPv6 is integrated in TORRENT as a transport protocol and the IP Security Protocol (IPsec) is used as a service for securing the data between the Residential Gateway (RG) and the Local Access Point (LAP). Investigations of various authentication and key agreement schemes have been carried out in the IPsec performance trials, as documented in [2].

\section{Background and Objectives}

It is a requirement of TORRENT to mutually authenticate the RG and the LAP; to provide users with secure access to TORRENT services; and also to authenticate users accessing the system e.g. to make changes to the user profile and user preferences (service subscription, QoS, cost, etc.) It foreseen that if a hardware (HW) token were used as key holder of the authentication exchange, then this HW token could also be used for user authentication (of the RG user) and eCommerce applications. The same token could be used for authentication and key exchange for an IPsec VPN tunnel service. Certificates and associated private keys for authentication and encryption of agents and agent communication could also be stored on the HW token. In fact, certificates and associated private keys for the services of user authentication, electronic signature, and encryption can be reused by TORRENT's agent based Service to Resource Management system for authentication of the user agents. Therefore, public key techniques were explored early in the TORRENT project.

\subsection{Assumptions}

We make the following general assumptions about the TORRENT system:

- The RG is vulnerable

- The link between the RG and LAP is vulnerable.

- The user access to the user profile interface is vulnerable.

- The link (not shown) between service domains (e.g., LAP to LAP) is vulnerable.

\subsection{Security Objectives}

The authentication requirements for the TORRENT system have been determined by a threat analysis. It was determined that the threats of masquerading by LAP or RG can be mitigated by mutually authenticating the LAP and the RG. Authentication is important e.g. to ensure that the authorised customer behind an RG is getting the QoS that was requested, to reduce the likelihood of fraud and also as a baseline for avoiding repudiation of messages e.g. payments. Users and providers of networks and services will thus benefit from this security service. A scheme for providing this will be presented later in the paper.

Authentication of agents to each other in the agent system is also required to prevent manipulation of traffic sent between agents. Authentication of agents is addressed in [4]. 


\subsection{Enabling Quality of Service}

Authentication alone will not guarentee that the customer receives the QoS that was requested. However, assuming that QoS mechanisms are in place to maintain and control the quality of service, mutual entity authentication is an important countermeasure to a number of threats to QoS provisioning.

For example, if authentication of the RG to the LAP is insufficient, an attacker may make a distributed attack from many false RGs allocating a substantial number of QoS enabled flows, which binds up the resources and degrades the performance for authorised user flows. An important mitigating measure to counter this attack is mutual authentication of the RG and the LAP. In the case of the TORRENT project, the user may select a level of QoS on a per service basis via a GUI hosted on the LAP. Sufficient authentication of the user for access to the GUI (and integrity protection of the communication on the link between the RG and the LAP) is required to prevent unauthorised users from masquerading as authorised customers in order to obtain a higher level of QoS without paying for it.

\section{Authentication and Key Agreement Based on Public Key Techniques}

In this section we will discuss the case for public key authentication techniques for network access.

\subsection{Background}

Historically, authentication for network access has been done using shared secret techniques and this has proven to be scalable. For ISDN and now ADSL the residential user is issued a terminal per service (NT box for ISDN, ADSL modem for ADSL). ADSL modem authentication is at best, password based and open to dictionary attacks when the password is small or insufficiently random. Requirements have been lax: it is well known that most ADSL modems can be hacked without requiring sophistication [5]. 2G and 3G mobile telecommunication systems also use secret key techniques for network access.

As the number of access networks increases scalabity issues become more significant. In GSM, for example, scalability is achieved using brokers and roaming agreements. A small operator has an agreement with a roaming broker, which establishes roaming agreements with a lot of other operators. It can be argued that as the number of operators and roaming agreements grows significantly, then these bilateral agreements may be inefficient and costly to maintain. But essentially, scalability issues alone do not provide a strong argument for the case for public key techniques for network access.

Public key techniques were ruled out early on in the $3 \mathrm{G}$ design and standardisation process as these were considered too complex and seemed to require too much computational overhead. Since then, however, public key based 
mechanisms have been successfully implemented in the GSM SIM card for e.g. mCommerce [9] and in smartcards [6], demonstrating that computational overhead is no longer an issue.

The strong arguments for public key techniques are scalability and to some extent mobility (of the user to choose freely between networks and service providers), and the elegance of the reusability of public key techniques for a multitude of services such as authentication, and key exchange, notary public services, eCommerce, mCommerce, and electronic signatures. Use of public key techniques is also motivated in part by stronger requirements for user anonymity - public key authentication offers possibilities for providing strong user identity and location confidentiality as the user id and location information does not need to be transmitted in the clear over the network. For example, the public key of the authorised receiving party can be used to encrypt the identity and other private information belonging to the user.

\section{Authentication in TORRENT}

In this section we will propose a scheme for public key authentication for securing network access from the user of the RG to the LAP.

The LAP is fitted with a hardware key container with a (possibly several) server certificate(s) and associated private key(s). A smartcard as certificate and key container is inserted in the RG. The user behind the RG has a relationship with a trusted third party (Òcertificate validator/brokerÓ).

At least one X.509 certificate and associated pair of keys are stored on the smartcard for authentication and encryption purposes (actually, the public key is contained in the certificate, while the private key is not). The trusted third party's public key certificate is also stored on the smartcard to enable validation of foreign incoming certificates.

The RG user Smartcard contains a certificate binding the RG user to a public key suitable for both encryption and signature verification (or it contains two separate certificates). The LAP has a certificate binding the LAP operator to certificate issued by a Certificate Authority (CA). Both must have X.509 certificates that can be validated by a trusted third party, which we call the Certificate Validation Clearinghouse/Broker (CVC). The CVC functions as a trusted third party and has an agreement with the CA that issued the certificate to the LAP allowing it to perform certificate validation services on behalf of the $\mathrm{CA}$, and it also has such an agreement with the CA that issued the certificate(s) to the RG user. The CVC public key is installed on the RG user smartcard a priori. Note, it is feasible that the RG user may have the public keys of several CVCs installed on their smartcard.

The aim is to mutually authenticate the RG and the LAP using public key techniques. The motivation for this is the case that the user of the RG does not have to be bound to a Service Provider or Operator (by a subscription) but is free to shop around for network access and services. In this case, shared secret 
keying techniques are not appropriate. It should be noted that this algorithm can be applied to both wired and non-wired network access.

Use of public key techniques for network access has been studied in the SHAMAN project [7] and two methods for authentication and key agreement using public key techniques are described. The protocol presented in this paper is different from those presented by SHAMAN. In the method described in SHAMAN the mobile node has a subscription to a home operator, and must establish network access with an access network, which has an agreement with the home operator or a roaming broker. This network access point (e.g. LAP) has a number of pre-installed public key certificates (signed by each trusted third party or home network with which it has an agreement). The access network sends the appropriate one to the node (e.g. RG) and this is used by the node to assure the node that a roaming agreement exists with the home network.

Similarly to SHAMAN, public key techniques are used to mutually authenticate the LAP to the RG and the RG to the LAP using the the CVC. In the TORRENT case, however, the user does not necessarily have a relationship/subscription to a home network, nor does the LAP have to have an agreement with other access network operators. It is the CVC's public key that is pre-installed in the RG user's smartcard and also on the LAP, and which is used in the validation process. The CVC can function as a broker and a clearinghouse.

The CVC validates the RG user's certificate on behalf of the Certificate Authority for the LAP, and the LAP's certificate on behalf of the RG. . How this validation is performed is outside of the scope of this paper: a thorough explanation can be found in 3 .

Once the certificate validation process is completed, the type of payment for services (e.g., bandwidth, QoS, VPN) can be agreed using the preferred payment method such as credit card, online banking, billing directly based upon some method associated with the smartcard, etc. (again, the exact method used is outside of the scope of this paper).

\section{Protocol RRW Strong Two-Way Entity Authentication}

The basic protocol involves $A$ (the RG User Smartcard), $B$ (the LAP), and $T$ (the CVC server). The initial checking of certificates incorporates the X.509 protocol for strong Three-Way Authentication 1].

At outset the RG User Smartcard contains at least one public key pair suitable for both encryption and signature verification and in accordance with X.509 standards. The RG user's Smartcard must also acquire (and authenticate) the CVC encryption public key a priori.

The LAP has its public key pair for signature and encryption. The CVC server has a public key pair for signature and encryption. The CVC has an agreement with the CA that issued the certificate to the LAP, allowing the CVC to validate on behalf of the CA which issued the LAP certificate. The CVC also has an agreement with the $\mathrm{CA}$ that issued the card to the user. 
SUMMARY: RG User Smartcard interacts with a Trusted CVC server and LAP.

RESULT: mutual entity authentication.

\section{Notation}

$A$ denotes the RG User Smartcard.

$B$ denotes the LAP

$T$ denotes the CVC server.

$I_{T}$ denotes the identification of the $\mathrm{CVC}$ so that the authentication request can be sent to the correct CVC.

$P_{X}(y)$ denotes the result of applying $X$ 's encryption public key to data $y$. $S_{X}(y)$ denotes the result of applying $X$ 's signature private key to data $y$. $r_{X}$ denotes a random number generated by $X$.

$d_{X}$ denotes a random number generated by $X$.

Cert $_{X}$ is a certificate binding party $X$ to a public key suitable for both encryption and signature verification. Remark: A good practice is to avoid using the same cryptographic key for multiple purposes.

cert $_{X}$ ok denotes the "Cert C $_{X}$ has been validated" message.

\section{System Setup}

(a) Each party has its public key pair for signature and encryption.

(b) The encryption public key of the CVC is installed on both the the RG user Smartcard and the LAP a priori.

(c) The CVC server has an agreement with the Certificate Authority (CA) that issued $A$ 's public key certificate(s) allowing the $\mathrm{CVC}$ to validate $A$ 's certificate on behalf of the CA, and similarly with the Certificate Authority that issued $B$ 's public key certificate.

3. Protocol messages

$$
\begin{aligned}
& A \rightarrow B: \operatorname{Cert}_{A}, r_{A}, S_{A}\left(r_{A}\right) \\
& A \leftarrow B: \operatorname{Cert}_{B}, S_{B}\left(r_{A}, r_{B}\right), r_{A}, r_{B} \\
& A \rightarrow B:\left(r_{B}, S_{A}\left(r_{B}\right)\right), P_{T}\left(\operatorname{Cert}_{A}, \operatorname{Cert}_{B}, d_{A}, r_{A}, r_{B}\right), I_{T} \\
& B \rightarrow T: P_{T}\left(\operatorname{Cert}_{A}, \operatorname{Cert}_{B}, d_{A}, r_{A}, r_{B}\right), S_{A}\left(r_{B}\right) \\
& A \leftarrow T: P_{A}\left(\operatorname{cert}_{B} \text { ok, } S_{T}\left(d_{A}\right)\right) \\
& B \leftarrow T: P_{B}\left(\operatorname{cert}_{A} \text { ok, } S_{T}\left(r_{B}\right)\right)
\end{aligned}
$$

4. Protocol actions

a) $A$ generates $r_{A}$, signs it using $A$ 's own private key, and sends to $B$ message (1).

b) $B$ extracts $A$ 's public key from $\operatorname{Cert}_{A}$ and uses this to check the signature on the data $r_{A}$. If the check succeeds, $B$ is satisfied that $A$ 's posesses the private key corresponding to the certificate $\operatorname{Cert}_{A}$, but not that $B$ and $A$ are authorised to communicate. 
c) $B$ generates $r_{B}$, signs the concatenation of this and $r_{A}$ and passes message (2) back to $A$.

d) $A$ extracts $B$ 's public key from $\operatorname{Cert}_{B}$ and uses this to check the signature on the data $\left(r_{A}, r_{B}\right)$. If the check succeeds, $A$ is satisfied that $B$ possesses $\operatorname{Cert}_{B}$ and its corresponding private key, but not that $A$ is permitted to communicate with $B$.

e) $A$ signs the message $r_{B} . A$ also generates $d_{A}$, a new challenge value to be answered by the CVC. $A$ encrypts data for the CVC server $T$ containing $\operatorname{Cert}_{A}, \operatorname{Cert}_{B}, r_{A}, r_{B}$ and $d_{A}$. This encrypted message is sent along with $\left(r_{B}, S_{A}\left(r_{B}\right)\right)$ and the plaintext CVC identification $I_{T}$ to $B$ (for relaying to $T)$.

f) $B$ checks the signature on the data $r_{B}$, then $B$ uses the cleartext identifier $I_{T}$ in message (3) to relay (4) to $T$. Before relaying the message, $B$ appends $S_{A}\left(r_{B}\right)$, ( $A$ is sending $r_{B}$ inside the encrypted message).

g) $T$ decrypts (4) using its private decryption key. $T$ validates Cert $_{A}$ and $\operatorname{Cert}_{B} . T$ extracts $A$ 's public key from $\operatorname{Cert}_{A}$ and uses it to verify that $S_{A}\left(r_{B}\right)$, as appended by $B$ is a valid signature of the $r_{B}$ value which was encrypted by $A$. This check prevents $A$ from exchanging one certificate with $B$ initially, but sending a different one to $T$ for authentication.

h) $T$ signs $r_{B}$ and $d_{A}$, and sends the messages in (5) and (6). Each message is encrypted using the recipient's public key.

i) $A$ decrypts (5) and verifies $T$ 's signature on the challenge $d_{A}$. If this signature is valid, $A$ declares authentication of $B$ successful.

j) $B$ decrypts (6) and verifies $T$ 's signature on the challenge $r_{B}$. If this signature is valid, $B$ declares authentication of $A$ successful.

Note: Upon authentication, $A$ and $B$ may proceed directly with a keyexchange for IPsec. Key exhcange was deliberately decoupled from the authentication process to allow operators to offer VPN as a value-added service if they wish.

\subsection{Periodic Validation}

To prevent redirection of the communications channel once authentication has been completed, it is necessary to periodically ensure that the authenticated parties are still in control of the communications channel. The method used here is a periodic "challenge-response" from LAP to the Card in the RG.

If the LAP does not receive a signed response from the card within a reasonable amount of time, the LAP will cease routing traffic to or from the RG containing the non-responsive card. Conversely, if the Card does not receive a challenge from the LAP within a reasonable time, it will disable the RG interfaces on the assumption that the LAP has either failed or has been compromised. 


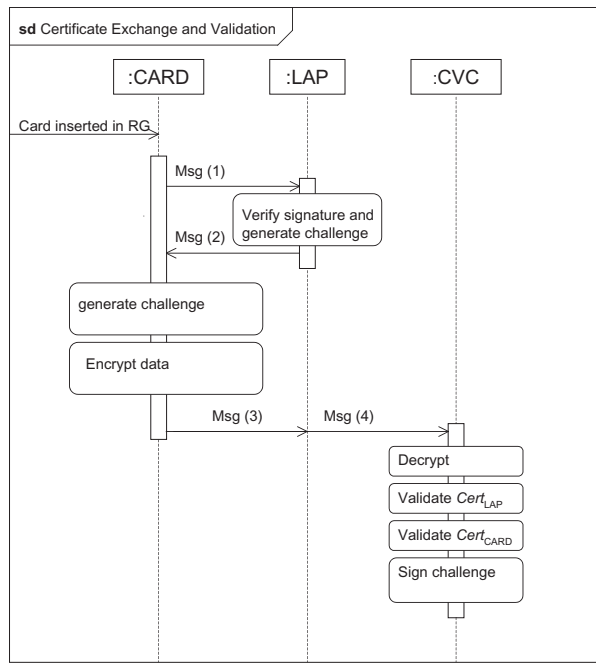

(a)

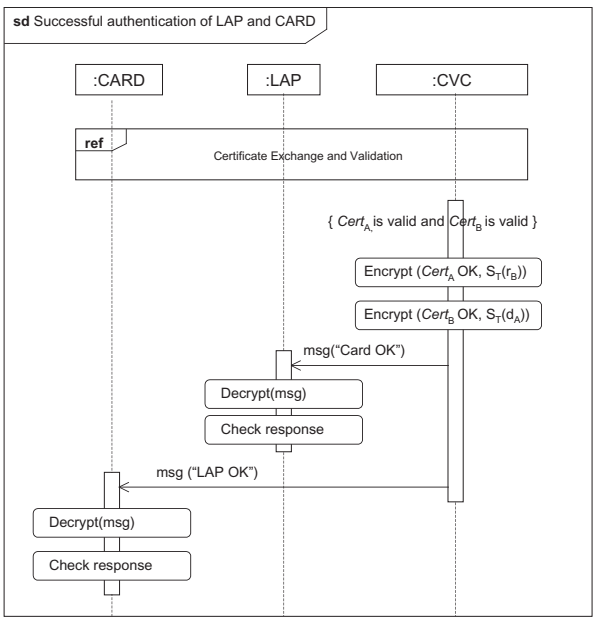

(b)

Fig. 2. Authentication Sequence: (a) initial LAP/Card certificate exchange, (b) sucessful authentication process

\section{Sequence Diagrams}

Figure 2 shows the authentication progression. The two alternative sequences in Figure 3 show possible deviations in authentication progression, depending on the validity of the LAP or RG smartcard holder's certificates.

\section{$7 \quad$ Feasibility}

The greatest barrier to implementation of this system is the ability of the smart card processor to perform the necessary encryption and decryption operations.

The protocol presented here requires the smart card to perform one signature, one encryption, one decryption and one signature validation. Of these, the encryption (of the combined certs and challenges message) involves by far the largest amount of data. These operations must be performed on-card, as the RG in which the card sits is a non-trusted system.

\subsection{Encryption Algorithm}

Because of the limited computational power of the smart card processor, a computationally-light, but still secure encryption scheme is required. Elliptic Curve Cryptography (ECC) [15] offers strength at least equal to the more widelydeployed RSA. However, ECC requires shorter key lengths and lower computational load[16], and as such would be an ideal candidate for use in the system described here. 


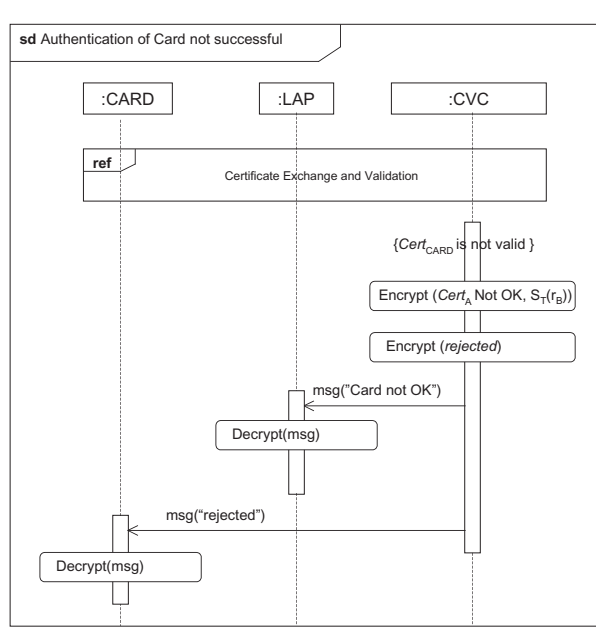

(a)

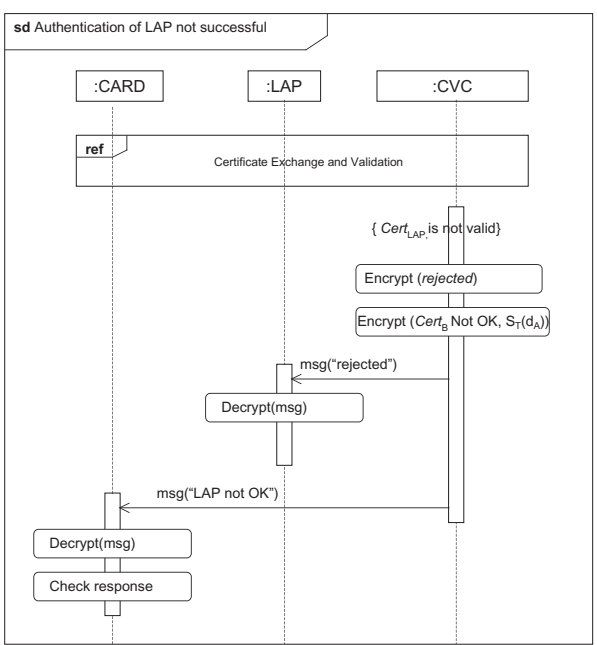

(b)

Fig. 3. Sequences for failed authentication process - (a) invalid card, (b) invalid LAP

\subsection{Smart Card Performance}

The encryption and decryption operations performed by the card are not timecritical: because authentication is not performed regularly, a time of up to three seconds from card insertion (a similar performance to most pay TV systems) is acceptable and easily achievable.

Current microprocessor smart cards can already be used for key generation and encryption using ECC techniques, and so are well able to perform the encrypt/decrypt operations required by the protocol presented here. 17] [18.

\subsection{Threat Analysis}

The primary weakness in this system, as in all trusted-party systems, is the integrity of the CVC server. However, assuming that the operator of the CVC Server takes adequate measures to protect it from subversion, destruction or replacement, a potential attacker is left with only three other targets: the smart card, the RG, and the LAP. Each of these is examined in turn.

Smart Card. The Smart card is a closed computer whose only means of communication with the RG is via a simple serial data link [19]. The firmware running on the smart-card card CPU controls what information is sent along this serial link to the RG.

The smart card private key and public key pair are written into on-card read-only memory during manufacture. The software on the smart card will never cause the private key to be sent out of the card, and there is no other way 
to access the on-card memory without resorting to industrial disassembly of the card chip.

Messages from the smart card to the LAP and CVC are encrypted on the card before being passed to the RG for transmission. The exception to this is the initial exchange of certificates between smart card and LAP, when the smart card sends its X.509 certificate as plaintext. However, the only information of consequence in this certificate is the card's public key, disclosure of which does not compromise the safety of the card's private key. 13.

RG. The RG is envisioned as a mass-produced, dedicated computer system, based on open-standards. As such, the RG is susceptible to substitution: a malicious user can create their own fraudulent RG using a general purpose PC and a smart-card interface. It is precisely for this reason that the protocol as presented here assumes that the RG is a hostile party in the system.

No critical messages are passed to the RG without first being encrypted. The RG can choose not to forward these messages, but this will result in a denial of service, as the LAP will not enable the RG to LAP link until authentication is complete.

It is conceivable that a tampered RG could be re-programmed to perform authentication once with a legitimate card inserted, and then remain on-line indefinitely, regardless of the presence of the smart card. The periodic validation scheme 5.1 is designed to defeat this attack: without a legitimate card in the $\mathrm{RG}$ to answer the challenge of the LAP, the RG will quickly be disconnected from the network.

LAP. The LAP is a dedicated computer system owned and maintained by the network operator, and located on their property. As such, the LAP is a less attractive target for direct attack than the RG or smart card. However, where a shared-medium network (e.g., wireless) serves the LAP and RGs, the risk of LAP substitution arises.

In order to defeat a user who sets up a fraudulent LAP and replays the genuine LAP's contributions to the authentication protocol, the genuine LAP passes a random challenge message to the the smart card during the initial LAP/Card certificate exchange.

If a LAP's private key is somehow disclosed, the compromised LAP can be issued with a new key pair, and the CVC server can reject any messages signed with the old, compromised, private key. No change is necessary to the smart cards, as they do not store LAP information.

\section{Comparison with Existing Schemes}

It was our intention to use existing, open-standard protocols and technology wherever possible. For this reason, the protocol presented here incorporates the X.509 Strong Three-Way mutual Authentication protocol[1], and extends it for use with a trusted third party. 
The proposal is similar to Kerberos 12 in that it involves a party $A$ interacting with a trusted server $T$ and a party $B$. However, The encryption algorithm of Kerberos is symmetric, and public key techniques are not involved. A ticket is generated which allows $A$ to re-use the ticket for multiple authentications to $B$ without involving $T$.

In our approach, multiple authentications are not necessary. The periodic validation function (5.1) allows the RG and LAP to communicate over long periods of time without requiring re-authentication. Once authentication is finished, the Card still keeps listening on a socket for an occasional "heartbeat" request from the LAP/Authentication server. So even if the card is removed without being able to send the "Disable Services" message to the RG (or if the $\mathrm{RG}$ has been tampered with to ignore this message), the LAP will quickly realise the card is no longer in the RG, and will stop routing the RG's traffic.

\section{Re-usability of the Certificates and Keys}

In this section we give a few examples of how the certificates and keys can be reused for securing other functions of the TORRENT system, for user authentication, application security, eCommerce, etc.

As explained above, the customer behind the RG has a smartcard inserted in the RG. The smartcard contains at least one X.509 certificate and associated asymmetric keys. The certificate belongs to the customer and is linked to the customer profile database. Depending on the certificate policy, the customer may have to present themselves in person to the $\mathrm{CA}$ to register and receive the certificate and keys.

The card and accompanying certificate(s) and keys may be used for key exchange when setting up an IPsec VPN to securing the access link between the RG and the LAP [2]. Using Qualified Certificates the smartcard may even suitable for creating electronic signatures [14] 20].

In a system where users can make changes to their profile on-line (e.g., QoS requests, billing details, etc.), the X.509 certificate can serve as authentication to these services. If the operator requires electronic signature of requests from users, the user can also sign them electronically at this point, provided that the card contains a certificate that can be used for electronic signature. It can also be foreseen that pay-per-use services can be provided using this scheme and that the user can provide an electronic signature for payment purposes.

\section{Conclusion}

\subsection{Discussion}

In this research we focus on mutual authentication procedure between the customer's residential gateway and the Local Access Point using a proposed authentication protocol that combines techniques that are already proven to be reliable (X.509 certificates, smartcards, public key cryptography). The motivation comes 
from a requirement of the TORRENT IST project for a method of mutually authenticating a residential gateway (in the home) and a local access point (in the operator's premises). Bearing in mind that with future multi-service residential access networks, users may have — or demand - the facility to choose between several network operators and service providers.

The authentication requirements of the different operators and providers can lead to a situation where the user has to keep track of several combinations of usernames and passwords which becomes much more difficult to manage as time progresses. The process described in this paper proposes a scheme in an attempt to solve these issues for the TORRENT project and other deployments of LAP/RG types of systems. The process can also be applied to authenthicate to, and access mobile access networks, e.g. for use in $3 \mathrm{G}$ and future networks where the smart card may be used for authentication to many services.

\subsection{Related Work}

Use of public key authentication and key exchange for network access has been studied in the IST-SHAMAN project [7]. The first method proposed by SHAMAN is described in 4 and requires each network access point (e.g. LAP) to have a number of pre-installed public key certificates (signed by each trusted third party or home network with which it has an agreement). As the LAP operates in a multi-provider environment, this scheme could quickly lead to a situation for which the LAP is overloaded by certificate processing.

Our method does not require any pre-existing agreement between the users and the networks they attempt to join, and pre-installation of home network operator certificates on the LAP is also not required.

The second method described in SHAMAN differs also from our work in that it does not require client authentication. This method is concerned with providing anonymous access to the network based on immediate payment for services and therefore does not require authentication of the client. This method is purely concerned with authenticating the network.

The WLAN Smartcard Consortium[1], which was established in February 2003 , is working towards defining specifications for world-wide access to WLAN networks using smartcard security in order to provide privacy, roaming and related capabilities. As this group is recently established, the specifications are not publicly available at this time. It is projected by the Consortium that they will be available by the end of the 2003 .

Similarly, the ETSI AT NGN@Home has announced plans for work on a deliverable entitled "Access and Terminals (AT); Home Area Networks and the support of Next Generation Services; Part 6: Security and Copyright issues" [10]. The intention is to outline the security and copyright (including Digital Rights Management and Privacy) issues related to the support and delivery of Next Generation Services and applications both to and within a Home Area Network. 


\subsection{Future Work}

Future work will include schemes for using the Smartcard to gain secure access to a service at a chosen QoS class. Methods for protection of personal privacy will also be investigated. In the age of full IPv6 deployment, it is envisioned that protection of personal privacy can be made much easier. With IPv6, and certificates, IP addresses can be public, but the certificate, coupled with public key techniques, can be used to govern what traffic is allowed in, and what traffic should be prohibited. Only users with pre-approved certificates can reach (and communicate with) the user at the destination IP address.

Acknowledgements. The research on which this paper reports has partly been supported by the IKT 2003 project SARDAS (15295/431) funded by the Research Council of Norway; and the European Union-supported TORRENT project (IST-2000-25187).

\section{References}

1. Menezes, A. J, P. C. van Oorschot, S. A. Vanstone, Handbook of Applied Cryptography, CRC Press, Florida, 1997

2. Ronan J, Malone P, Ó Foghlú M, Overhead Issues for Local Access Points in IPsec enabled VPNsÓ, IPS Workshop, Salzburg, February 2003. Retreived: 3. April, 2003 from http://www.ist-intermon.org/workshop/papers/09_01_vpn-overhead.pdf

3. Ølnes J, Trusted Certificate Validation Services - Breaking the PKI Deadlock, jon.olnes@validsign.com, http://www.validsign.com, 10. October 2002

4. Houmb, Siv Hilde. Security Issues in FIPA Agents, paper in progress.

5. Cert Advisory CA-2001-08, Multiple Vulnerabilities in Alcatel ADSL Modems, 12. April 2001. Retreived: 3. April, 2003 from http://www.cert.org/advisories/CA-2001-08.html

6. Schlumberger, Schlumberger Smart cards Cryptoflex Home Page, Retreived: 3. April, 2003 from http://www.cryptoflex.com/index.html

7. IST-SHAMAN Deliverable D09: ÒDetailed Technical Specification of Security for Heterogeneous AccessÓ, June 2002

8. TORRENT (Technology for a Realistic End User Access Network Test-bed), IST2000-25187. http://www.torrent-innovations.org

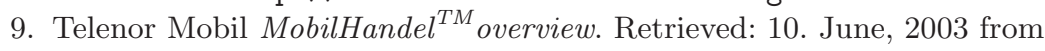
http://telenormobil.no/tjenester/mobilhandel/index.jsp

10. ETSI AT MGN@Home Access and Terminals (AT); Home Area Networks and the support of Next Generation Services; Part 6: Security and Copyright issues, work in progress. Retrieved 10. June 2003 from http://portal.etsi.org/Portal_Common/home.asp

11. WLAN Smartcard Consortium, homepage. Retrieved: 10. June, 2003 from http://www . wlansmartcard.org/.

12. Neuman B C and Ts'o T, "Kerberos: An Authentication Service for Computer Networks", IEEE Communications 32(9),pp 33-38. September 1994

13. Diffie $\mathrm{W}$ and Hellman $\mathrm{M}$ E, "New directions in cryptography", IEEE Transactions on Information Theory 22(1976), pp 644-654 
14. EU Directive 1999/93 on Electronic Signatures ETSI TS 111456 for Qualified Certificates

15. Satoh T, Araki K, Miura S. "Overview of elliptic curve cryptography", Proc. PKC'98, LNCS 1431,pp. 29-49, Springer-Verlag, 1998.

16. Gupta V, Gupta S and Chang S Performance Analysis of Elliptic Curve Cryptography for SSL ACM Workshop on Wireless Security (WiSe), Mobicom 2002, Atlanta, Georgia, USA September. 2002. Retrieved 10. June 2003 from http://research.sun.com/projects/crypto/performance.pdf

17. Aydos M, Yanık T, and Koç Ç, An High-Speed ECC-based Wireless Authentication Protocol on an ARM Microprocessor, Annual Computer Security Applications Conference, New Orleans, 2000. Retreived: 10. June, 2003 from http://acsac.org/2000/papers/24.pdf

18. Woodbury A, Efficient Algorithms for Elliptic Curve Cryptosystems on Embedded Systems, Worcester Polytechnic Institue, Massachusetts. Retrieved 10 June, 2003 from http://www.wpi.edu/Pubs/ETD/Available/etd-1001101 -195321/unrestricted/woodbury.pdf.

19. International Organisation for Standardization, ISO/IEC 7816-3:1997: Information technology - Identification cards - Integrated circuit(s) cards with contacts Part 3: Electronic signals and transmission protocols, Geneva, 1997.

20. Santesson S et al, RFC 3039: Internet X.509 Public Key Infrastructure Qualified Certificates Profile, January 2001. Retreived: April 3, 2003 from http://www.ietf .org/rfc/rfc3039.txt 